Macedonian Pharmaceutical Bulletin, 66 (Suppl 1) 111 - 112 (2020)

Online ISSN 1857 - 8969

UDC: 665.585 .5

DOI: 10.33320/maced.pharm.bull.2020.66.03.055

Short communication

\title{
The presence and type of SAAs in shampoo-type preparations
}

\author{
Jasmina Bašić $^{1 *}$, Isidora Milanović ${ }^{1}$, Danijela Pecarski ${ }^{1}$, Dragana Dragaš Milovanović ${ }^{1}$ \\ ${ }^{I}$ Belgrade Academy of Professional Studies, Department Medical College of Professional Health \\ Studies, Cara Dušana 254, Zemun, Serbia
}

\section{Introduction}

SAAs (Surface Active Agents), surfactants or tensides, are amphiphilic substances of organic nature, which have the ability to be absorbed on the surface of particles, changing their surface tension. Thanks to their amphiphilic, polar-nonpolar dual nature, one part of their molecule is soluble in a particular liquid (the lyophilic part), whereas the other part is not soluble (the lyophobic part). The polar part of a molecule is called the head and is hydrophilic, whereas the nonpolar part is the hydrophobic tail soluble in fats. SAAs are substances present in washing agents, face and body care products, medications and parapharmaceuticals, food products, as well as textile, paint and oil production, and in many other products. Those SAAs have different roles: moisturizers, emulsifiers, solubilizers, foamers/antifoamers, rheologic modifiers, antistatic agents, lubricants (Schramm et al., 2003) so, they have a role of a detergent in cosmetic preparation (shampoos, liquid soaps); in cosmetic emulsions (lotions and creams), they have a role of an emulsifier; in toothpastes, gels, lotions, solutions, they have a role of solubilizers or moisturizers.

Cleanliness maintenance products primarily have a task to clean the skin and hair from impurities, natural secretes (sebum and sweat), dead cells and the largest number of microorganisms. We need to be very cautious because of potentially damaging the hydrolipid film of the epidermis due to an excessive use of washing agents. The shampoo acting mechanism implies the creation of micelles in water, which have an affinity for fat and oil components which also include different impurities on the skin and hair, sebum included. The long-term and excessive use of these agents may lead to the drying-out of the skin and hair; the natural $\mathrm{pH}$ of skin changes, and the lipid/protein ratio in the horny layer is disturbed by the loss of the fats which have a protective role.

\section{Materials and methods}

In the research, an analysis of the chemical composition of 10 types of the shampoo of foreign production and 10 types of the shampoo of the domestic production (Serbia) was carried out, and a review of the type and amount of the present surface active agents in the shampoos was made with a reference to the safety of their application and potential adverse reactions.

\section{Results and discussion}

In the analyzed shampoo-type preparations, two types of ingredients are differentiated: SAAs (10$30 \%$ ) with the HLB value from 13 to 15 , and different additive types. In shampoos the basic or

\footnotetext{
* basic.jasmina23@gmail.com
} 
primary SAAs are anionic, which serve to wash the vertex and hair, and auxiliary or secondary are amphoteric and nonionic, which improve the characteristics of the basic ones.

Topologically, SAA molecules consist of the compact polar head and the flexible nonpolar tail, which create micelles in a water solution in the selfaggregation process. Heads have a strong affinity for polar solubilizers, particularly in water (those are hydrophilic parts), whereas the nonpolar part of the molecule is a hydrophobic or lipophilic part soluble in fats. The polar group is represented by a heteroatom: oxygen, nitrogen, sulfur, phosphorus in the composition of the alcoholic, thiolic, etheric, amino-, carboxylic, ester, sulfate, phosphate functional groups. A hydrocarbon branched or linear alkyl (alkylbenzene) chain 8-18 $\mathrm{C}$ atoms in length, which can be substituted by the atoms of halogen represents the hydrophobic part of the molecule. (Salager, 2002) The choice of a polar group (ionic or nonionic), as well as the relative size of the polar head and the nonpolar tail, is significantly determined by the physical-chemical characteristics of tensides (Mandavi et al., 2008). Physicochemical characteristics are also determined by the degree of the branching of the chain and the position of the polar group in the molecule of a surfactant. (Holmberg et al., 2002).

Anionic tensides have a role to create a foam and remove impurities. Most frequently and in the greatest percentage, we found alkyl-sulfates and alkyl-ether-sulfates, in the shampoos. Besides, there are also amidetersulfates, methyl-taurides and protein condensation products. Sodium-laurylsulfate, sodium-xylene-sulfonate, sodium-laurethsulfate and sodium-lauryl-ether-sulfate are the most present in the analyzed shampoos.

Beside anionic tensides, there are also amphoteric, whose representatives are the four betaine groups: miranols (imidazole derivatives), $\mathrm{N}$ alkyl-beta-amino-propionates, N-alkyl-betaimindipropionates and betaines with amides of longchain acids. Cocamidopropyl betaine is most present in shampoos. Amphoteric tensides are known to have bactericidal characteristics and mildly act on the skin of the head, alleviating the irritating action of anionic SAAs. Coconut acid diethanolamide is most frequently found in the shampoos. Their significant role also implies the alleviation of the irritation caused by anionic tensides. The anionic surfactant group is most and in the greatest percentage present in shampoos because of its detergent features and good solubility in water. They are stable in an alkaline environment and are not compatible with cations because of the formation of complexes and a reduction in the surface activity. They are economically cost-effective, and their safety for humans and animals has been researched by the Cosmetic Ingredient Review.

\section{Conclusion}

Because of the diverse applications of SAAs, their distribution into the living environment is great. There is an aspiration to formulate "greener" SAAs, which will have a minimal influence on the living environment, low toxicity and ecological justification. New, green surfactants should be of a structure which will not be any threat to the health of users, without the mutagenic and carcinogenic potentials and a tendency to develop toxicity (Jessop et al., 2015).

\section{References}

Holmberg, K., Jönsson, B., Kronberg, B., Lindman, B., 2002. Surfactants and Polymers in Aqueous Solution. second edition. Chichester: Wiley \& Sons, Ltd.

Jessop, P.G.F., Ahmadpour, F., Buczynski, M.A., Burns, T.J., Green II, N.B., Korwin, R., Long, D., Massad, S.K., Manley, J.B., Omidbakthsh, N., Pearl, R., Pereira, S., Predale, R.A., Sliva, P.G., VanderBilt, H., Weller, S., Wolf, M.N., 2015. Opportunities for greener alternatives in chemical formulations, Green Chem. 17, 2664.

Mandavi, R., Sar, S.K., Rathore, N., 2008. Critical micelle concentration of surfactant, mixedsurfactant and polymer by different method at room temperature and its importance. Orient J. Chem. 24(2), 559-564.

Salager, J.L., 2002. Surfactants, Types and uses. FIRP Booklet no. E300A, University of Los Andes, Merido, Venezuela.

Schramm, L.L., Stasiuk, N., Marangoni, D.G., 2003. Surfactants and their applications. Annu. Rep. Prog. Chem. Sect. C. 99, 3-48.

Maced. Pharm. Bull. 66 (Suppl 1) 111 - 112 (2020) 